Brutzer, Alexandra; Kastrup, Julia; Kettschau, Irmhild

\title{
Ausbildungsberuf Hauswirtschafter/in. Profilschärfung der Ausbildung für ein zukunftsfähiges Beschäftigungsfeld
}

Haushalt in Bildung \& Forschung 7 (2018) 1, S. 3-21

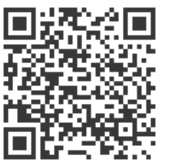

Quellenangabe/ Reference:

Brutzer, Alexandra; Kastrup, Julia; Kettschau, Irmhild: Ausbildungsberuf Hauswirtschafter/in.

Profilschärfung der Ausbildung für ein zukunftsfähiges Beschäftigungsfeld - In: Haushalt in Bildung \& Forschung 7 (2018) 1, S. 3-21 - URN: urn:nbn:de:0111-pedocs-190703 - DOI: 10.25656/01:19070

https://nbn-resolving.org/urn:nbn:de:0111-pedocs-190703

https://doi.org/10.25656/01:19070

in Kooperation mit / in cooperation with:

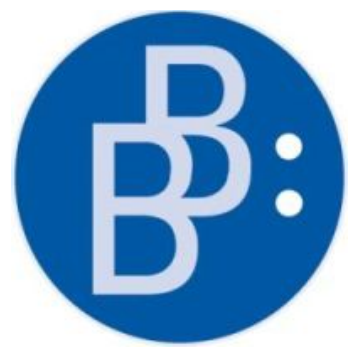

https://www.budrich.de

\section{Nutzungsbedingungen}

Gewährt wird ein nicht exklusives, nicht übertragbares, persönliches und beschränktes Recht auf Nutzung dieses Dokuments. Dieses Dokument ist ausschließlich für den persönlichen, nicht-kommerziellen Gebrauch bestimmt. Die Nutzung stellt keine Übertragung des Eigentumsrechts an diesem Dokument dar und gilt vorbehaltlich der folgenden Einschränkungen: Auf sämtlichen Kopien dieses Dokuments müssen alle Urheberrechtshinweise und sonstigen Hinweise auf gesetzlichen Schutz beibehalten werden. Sie dürfen dieses Dokument nicht in irgendeiner Weise abändern, noch dürfen Sie dieses Dokument für öffentliche oder kommerzielle Zwecke vervielfältigen, öffentlich ausstellen, aufführen, vertreiben oder anderweitig nutzen.

Mit der Verwendung dieses Dokuments erkennen Sie die Nutzungsbedingungen an.

\section{Terms of use}

We grant a non-exclusive, non-transferable, individual and limited right to using this document.

This document is solely intended for your personal, non-commercial use. Use of this document does not include any transfer of property rights and it is conditional to the following limitations: All of the copies of this documents must retain all copyright information and other information regarding legal protection. You are not allowed to alter this document in any way, to copy it for public or commercial purposes, to exhibit the document in public, to perform, distribute or otherwise use the document in public.

By using this particular document, you accept the above-stated conditions of use.

\section{Kontakt / Contact:}

peDOCS

DIPF | Leibniz-Institut für Bildungsforschung und Bildungsinformation Informationszentrum (IZ) Bildung

E-Mail: pedocs@dipf.de

Internet: www.pedocs.de

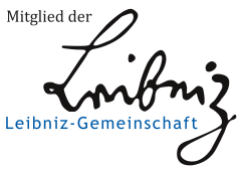


7. Jahrgang Heft 1 2018

$\bullet$

c

(1)

(1)

t)

$\frac{1}{c}$

종

진

$\stackrel{\frac{2}{d}}{>}$

$\bullet$

ธ

$\frac{0}{10}$

$\frac{1}{c}$

$\Phi$

ह

ฮั

क

+

$\stackrel{5}{ \pm}$

$\diamond$

$+$

$\frac{5}{0}$

而

ঠ
矛

$\frac{\Phi}{2}$

\section{Haushalt in \\ Forschung}

\section{Berufliche Bildung - Modernisierung von} Strukturen und Curricula

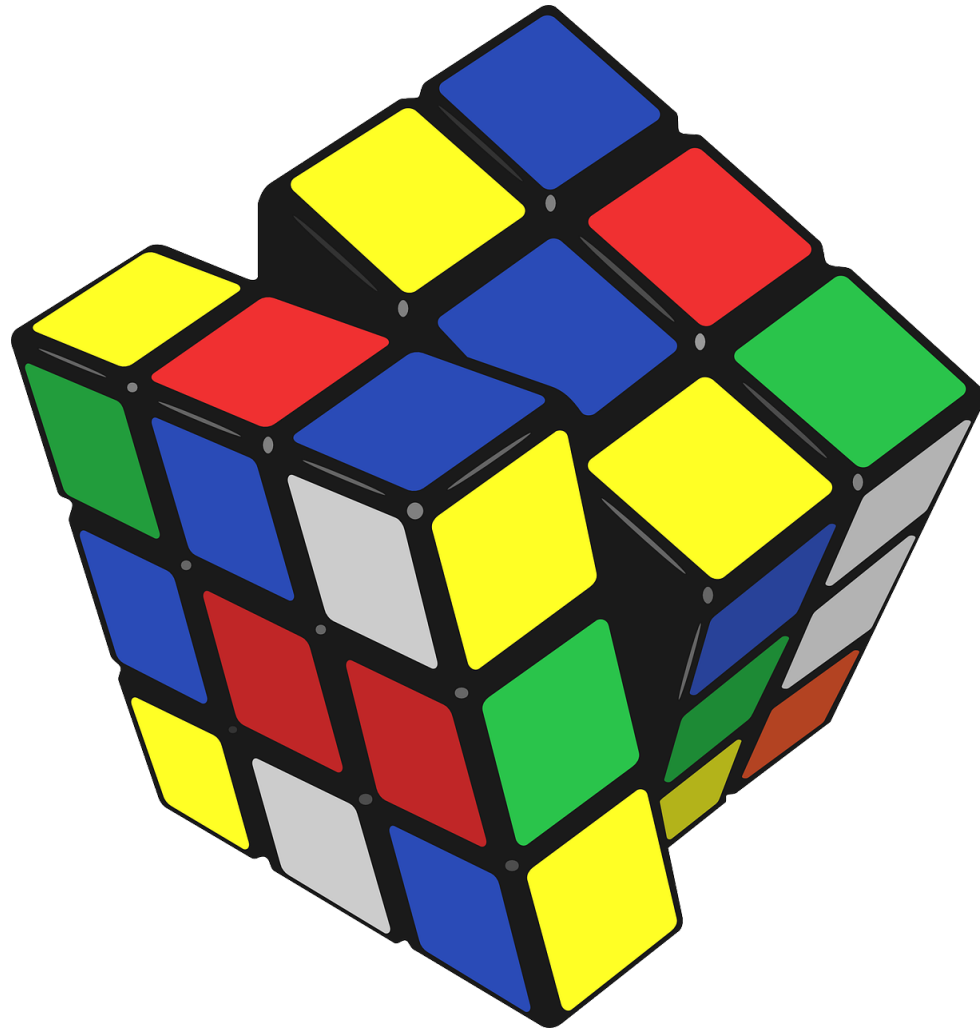

ISSN 2193-8806 
Inhaltsverzeichnis |

Julia Kastrup

Editorial

Alexandra Brutzer, Julia Kastrup \& Irmhild Kettschau

Ausbildungsberuf Hauswirtschafter/in - Profilschärfung

der Ausbildung für ein zukunftsfähiges Beschäftigungsfeld 3

Daniela Lund

Einfluss der Digitalisierung auf schulisches Lehren und Lernen in Lernfeldern -

Brauchen wir eine neue fachdidaktische Perspektive?.

Ursula Bordewick-Dell, Julia Kastrup \& Marie Nölle-Krug

Experimente im Berufsfeld Ernährung und Hauswirtschaft - ein Konzept

für die berufliche Lehramtsausbildung 38

Rita Brand

Struktur und Nutzen einer vollzeitschulischen Ausbildung in einer

Berufsfachschule.

Sarah Loy

Curriculare Verankerung der Beruflichen Bildung für nachhaltige Entwicklung am Beispiel eines Bildungsplans in NRW.

Maraike John \& Kerstin Starke

Berufliche Bildung für nachhaltige Entwicklung im Konditorenhandwerk - eine

Handreichung zur Modernisierung von Curricula.

Stephanie Grundmann, Karin Groth \& Nina Langen

Vorschläge zur Überwindung des Theorie-Praxis-Gap in der universitären

Ausbildung der beruflichen Fachrichtung Ernährung und Hauswirtschaft. 95 


\section{Ausbildungsberuf Hauswirtschafter/in - Profilschärfung der Ausbildung für ein zukunftsfähiges Beschäftigungsfeld}

Das Berufsbild der Fachkraft in der Hauswirtschaft weist neben einer Reihe profilhemmender Faktoren auch wichtige Profiltreiber auf, die im vorliegenden Beitrag herausgearbeitet werden und die es bei einer Novellierung der Ordnungsmittel der hauswirtschaftlichen Ausbildung zu berücksichtigen gilt. So besteht die Chance den Ausbildungsberuf auf Dauer attraktiver, moderner und zukunftsfähiger zu gestalten.

Schlüsselwörter: Hauswirtschaft, Neuordnungsprozess, Profiltreiber und Profilhemmer

\section{Einleitung}

Seit Ende des Jahres 2015 wird für den Ausbildungsberuf der Hauswirtschafterin/des Hauswirtschafters über eine Novellierung der Ordnungsmittel (Ausbildungsverordnung, Rahmenlehrplan) diskutiert, die letztmalig vor knapp 20 Jahren (1999) erfolgte. Auf Fachveranstaltungen und Workshops, initiiert von verschiedenen Akteuren (initial vor allem das Kuratorium der Deutschen Wirtschaft für Berufsbildung (KWB) als Vertreter der Arbeitgeberseite) werden Stärken und Schwächen, Potenziale und begrenzende Aspekte diskutiert. Ein Eckdatenentwurf zur Ausgestaltung des Berufsbildes liegt vor, der zwischen den Spitzenorganisationen der Sozialpartner, des KWB und den zuständigen Stellen beim Deutschen Gewerkschaftsbund (DGB) abgestimmt wurde. Somit steht einem Neuordnungsverfahren nichts mehr im Weg. Frühester Termin für das Inkrafttreten einer neu geordneten Ausbildungsordnung für die Hauswirtschaft wäre der 1. August 2020. Voraussetzung ist, dass bis Anfang November 2018 die Spitzenorganisationen der Sozialpartner, das KWB sowie der DGB auf Grundlage des Eckdatenpapiers zur Ausgestaltung der Ausbildungsordnung ein Antragsgespräch bei den zuständigen Fachministerien (Bundesministerium für Wirtschaft und Energie sowie Bundesministerium für Ernährung und Landwirtschaft) stattgefunden hat.

Grund für die Bestrebungen zu einer Neuordnung ist nicht allein die Tatsache, dass die letzte Novellierung weit zurückliegt und die Ordnungsmittel damit nicht mehr aktuell sind. Das Berufsbild der Hauswirtschaft ist mit einer Reihe an Herausforderungen, Problemen und stereotypen Ansichten konfrontiert, die das Profil der 


\section{Profilschärfung der Hauswirtschaft}

Hauswirtschaft schwächen (die sogenannten Profilhemmer). In einer Novellierung wird deshalb von der hauswirtschaftlichen „Szene“ die Chance gesehen, die Stärken und Potenziale der Hauswirtschaft (die sogenannten Profiltreiber) herauszuarbeiten, diese in den Ordnungsmitteln stärker als bisher zu berücksichtigen und damit das Berufsbild zu modernisieren und zukunftsfähig zu gestalten.

Im vorliegenden Beitrag wird deshalb die hauswirtschaftliche Berufsfamilie in den Blick genommen, um über ihre Analyse und die Betrachtung bisheriger Reformanliegen die profilhemmenden und -treibenden Faktoren herauszuarbeiten, die es aus Sicht der Autorinnen - im Rahmen des anstehenden Neuordnungsprozesses zu berücksichtigen gilt. Neben einer Zusammenfassung und Diskussion der wesentlichen Profiltreiber und Profilhemmer werden die für eine Novellierung entscheidenden Perspektiven aufgezeigt.

\section{Die hauswirtschaftliche Berufsfamilie}

Die hauswirtschaftliche Berufsfamilie bietet ein vielfältiges Beschäftigungsfeld, gekennzeichnet durch ein breites Spektrum an Aufgaben und Handlungsfeldern. Eine Vielzahl an Aus- und Weiterbildungsmöglichkeiten lässt das Berufsbild „Hauswirtschaft" (Fachkraftstufe) perspektivenreich erscheinen. Dennoch sinken die Ausbildungszahlen seit Jahren kontinuierlich und die Lernausgangslagen der Auszubildenden sind eher schwach. Die folgenden Ausführungen geben einen Einblick in Aufgaben und Kompetenzanforderungen der Hauswirtschaft; sie betreffen im Kern den Fachkraftberuf „Hauswirtschafterin/Hauswirtschafter“, greifen aber auch unterund übergeordnete Qualifikationsstufen auf.

\section{Beschäftigungsfeld und Leistungsbereiche}

Mögliche Einsatzgebiete der Hauswirtschaft sind (Brutzer, 2014, S. 132 f.; Wiener, Winge \& Zetsche, 2014, S. 16):

- Haushaltsnahe und familienunterstützende Dienstleistungen (z.B. Privathaushalte)

- Landwirtschaft (z.B. Gebäudereinigung, Produktvermarktung, Urlaub auf dem Bauernhof)

- Gemeinschaftsverpflegung (z.B. Kindertagesstätten, Schulen)

- Sozial- bzw. Altenpflege (z.B. Senioreneinrichtungen)

- Wellness (z.B. gesundheitsförderliche Lebensgestaltung, Ernährung)

- Tourismus (z.B. Gästebewirtung und -beherbung)

- Freizeit (z.B. Eventgestaltung).

Die damit verbundenen Aufgaben und Tätigkeiten sind unterschiedlich anspruchsvoll. Das Leistungsspektrum reicht von dispositiven und anleitenden Funktionen 


\section{Profilschärfung der Hauswirtschaft}

über organisatorische bis hin zu verfahrenstechnischen Aufgaben (siehe Abbildung 1).
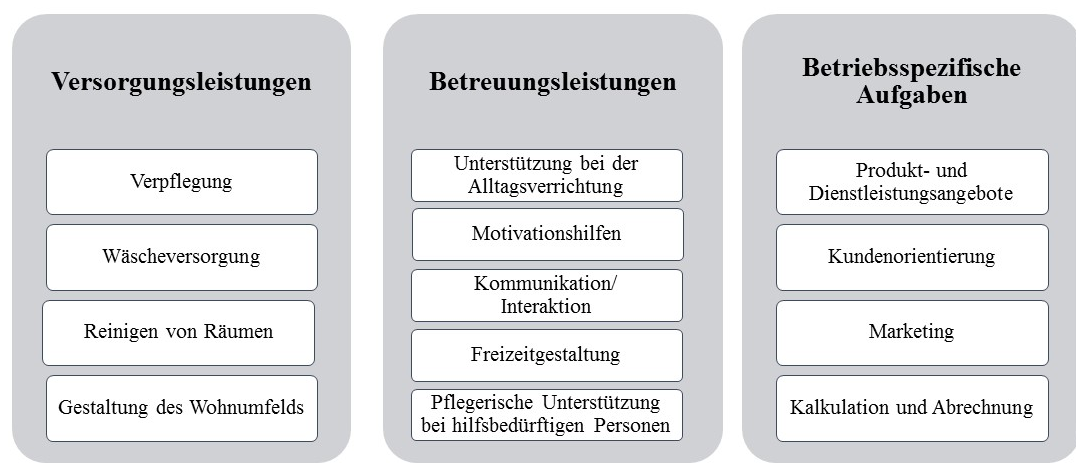

Abb. 1: Leistungsbereiche der Hauswirtschaft (Quelle: Brutzer, 2014, S. 219 in Anlehnung an Freese \& Toben-Vollmer, 2005; BAG HW \& dgh, 2012)

Um diese Aufgaben erfolgreich wahrnehmen zu können, braucht es entsprechende Kompetenzen auf definierten Niveaustufen, so wie diese auch im Deutschen Qualifikationsrahmen für lebenslanges Lernen (DQR) abgebildet werden. Die hauswirtschaftlichen Berufe wurden durch eine Arbeitsgruppe der Deutschen Gesellschaft für Hauswirtschaft e. V. (dgh) in der Systematik des DQR abgebildet (siehe Tabelle 1).

Tab. 1: Hauswirtschaftliche Berufe im DQR (Quelle: eigene Darstellung in Anlehnung an dgh, 2012, S. 18)

\begin{tabular}{l|l}
\hline Beruf/Niveau & Kernkompetenz \\
\hline $\begin{array}{l}\text { Stufe 1 und Stufe 2 } \\
\text { Berufsausbildungsvorbereitung }\end{array}$ & Nicht näher ausformuliert \\
\hline $\begin{array}{l}\text { Stufe 3 } \\
\text { Fachpraktikerin/ } \\
\text { Fachpraktiker }\end{array}$ & $\begin{array}{l}\text { Erfüllung von Anforderungen in einem überschaubaren, } \\
\text { z.T. offen strukturierten Tätigkeitsfeld der } \\
\text { hauswirtschaftlichen Betreuung und Versorgung }\end{array}$ \\
\hline $\begin{array}{l}\text { Stufe 4 } \\
\text { Hauswirtschafterin/ }\end{array}$ & $\begin{array}{l}\text { Planung und Bearbeitung fachlicher Aufgabenstellungen } \\
\text { in der hauswirtschaftlichen Versorgung und Betreuung } \\
\text { verschiedener Zielgruppen im umfassenden, sich } \\
\text { verändernden Tätigkeitsfeld }\end{array}$ \\
\hline $\begin{array}{l}\text { Stufe 5 } \\
\text { Fachhauswirtschafterin/ } \\
\text { Fachhauswirtschafter }\end{array}$ & $\begin{array}{l}\text { Planung und Bearbeitung umfassender fachlicher } \\
\text { Aufgabenstellungen hauswirtschaftlicher Versorgung } \\
\text { und Betreuung von Personen mit Hilfebedarf in einem } \\
\text { komplexen, spezialisierten, sich verändernden } \\
\text { Tätigkeitsfeld, insbesondere in der Alten- und } \\
\text { Behindertenhilfe }\end{array}$ \\
\hline
\end{tabular}




\section{Profilschärfung der Hauswirtschaft}

\begin{tabular}{l|l}
\hline Stufe 6 & Planung, Bearbeitung und Auswertung umfassender \\
Meisterin/Meister oder & Aufgaben- und Problemstellungen sowie \\
Betriebsleiterin/Betriebsleiter & $\begin{array}{l}\text { eigenverantwortliche Steuerung von Prozessen im } \\
\text { beruflichen Tätigkeitsfeld, die Anforderungsstruktur ist } \\
\text { komplex und verändert sich häufig }\end{array}$ \\
\hline Stufe 7 & Nicht näher ausformuliert \\
\hline
\end{tabular}

\subsection{Aus- und Weiterbildung}

Für die hauswirtschaftlichen Berufe besteht eine Vielzahl an Aus- und Weiterbildungsmöglichkeiten, die sich über alle Qualifikationsstufen erstrecken (siehe Abbildung 2). Sieht man von den Bildungsgängen der vorberuflichen Bildung ab (z.B. Berufsgrundbildungsjahr), ist zunächst die Ebene der Fachpraktikerinnen- und Fachpraktikerausbildung (gem. § $66 \mathrm{BBiG}$ ) zu nennen. Diese richtet sich an Menschen mit einer Behinderung (gem. § 2 SGB IX), denen es nicht möglich ist eine reguläre Ausbildung zu durchlaufen. Sie basiert auf landesspezifischen Erlassen, die sich an den Empfehlungen des Hauptausschusses des Bundesinstituts für Berufsbildung (BIBB) orientieren. Die Ausbildung dauert drei Jahre und findet in der Regel in überbetrieblichen Bildungsstätten statt. Der Zugang zu dieser Ausbildung erfolgt über eine Eignungsuntersuchung der Bundesagentur für Arbeit (Brutzer, 2014, S. 94).

Der Einstieg auf Fachkraftebene kann auf drei unterschiedlichen Wegen erfolgen. $\mathrm{Zu}$ nennen ist hier zum Ersten die reguläre dreijährige duale Ausbildung zur Hauswirtschafterin/zum Hauswirtschafter. Grundlage für die Ausbildung ist die Verordnung vom 30. Juni 1999 (BGBl. I S. 1495) sowie der dazugehörige Rahmenlehrplan (KMK-Beschluss vom 08. Juni 1999, BAnz. Nr. 234a vom 10.12.1999). Der erfolgreiche Abschluss eröffnet die Möglichkeit, sich anschließend in Fachschulen oder in Vorbereitungslehrgängen zur Meisterin/zum Meister der Hauswirtschaft oder zur Fachhauswirtschafterin/zum Fachhauswirtschafter weiterzubilden (ebd.).

Zum Zweiten besteht die Möglichkeit einen hauswirtschaftlichen Abschluss auf Fachkraftebene mittels vollzeitschulischem Bildungsgang zu erlangen. Es handelt sich hier um Ausbildungen ohne Ausbildungsvertrag bzw. betrieblichen Partner. Die erforderlichen Praxisanteile werden über die berufspraktische Ausbildung in den schuleigenen Praxisräumen sowie Betriebspraktika abgedeckt. Zu unterscheiden sind die einjährige Berufsfachschule, die ausschließlich eine Teilqualifikation vermittelt, die auf eine sich anschließende einschlägige Ausbildung in einem anerkannten Ausbildungsberuf angerechnet werden kann sowie die mehrjährige Berufsfachschule in deren Kontext vollqualifizierende Abschlüsse gem. Landesrecht erworben werden können. Die Ausgestaltung der Bildungsgänge unterscheiden sich je nach Bundesland (ebd.). 


\section{Profilschärfung der Hauswirtschaft}

Um zum Berufsabschluss zu gelangen ist drittens die Externenprüfung gem. $\S 45$ Abs. 2 BBiG möglich. Personen, die eine mindestens viereinhalbjährige einschlägige Tätigkeit in der Praxis oder eine entsprechende berufliche Handlungsfähigkeit nachweisen, können generell von den zuständigen Stellen zu einer Externenprüfung zugelassen werden. Im hauswirtschaftlichen Bereich wird auch die Tätigkeit in der Führung eines Mehrpersonenhaushalts (z.B. eigener Familienhaushalt) als berufliche Erfahrung im Sinne des BBiG gewertet, was zu einer Externenprüfung führen kann. Für den Ausbildungsberuf Hauswirtschafterin/Hauswirtschafter belief sich der Anteil der Externenprüfung an allen durchgeführten Abschlussprüfungen auf rund $47 \%$. Im Vergleich: der Durchschnittswert für alle durchgeführten Externenprüfungen beträgt berufsfeldübergreifend nur knapp $6 \%$ (BIBB, 2017, S. 175).

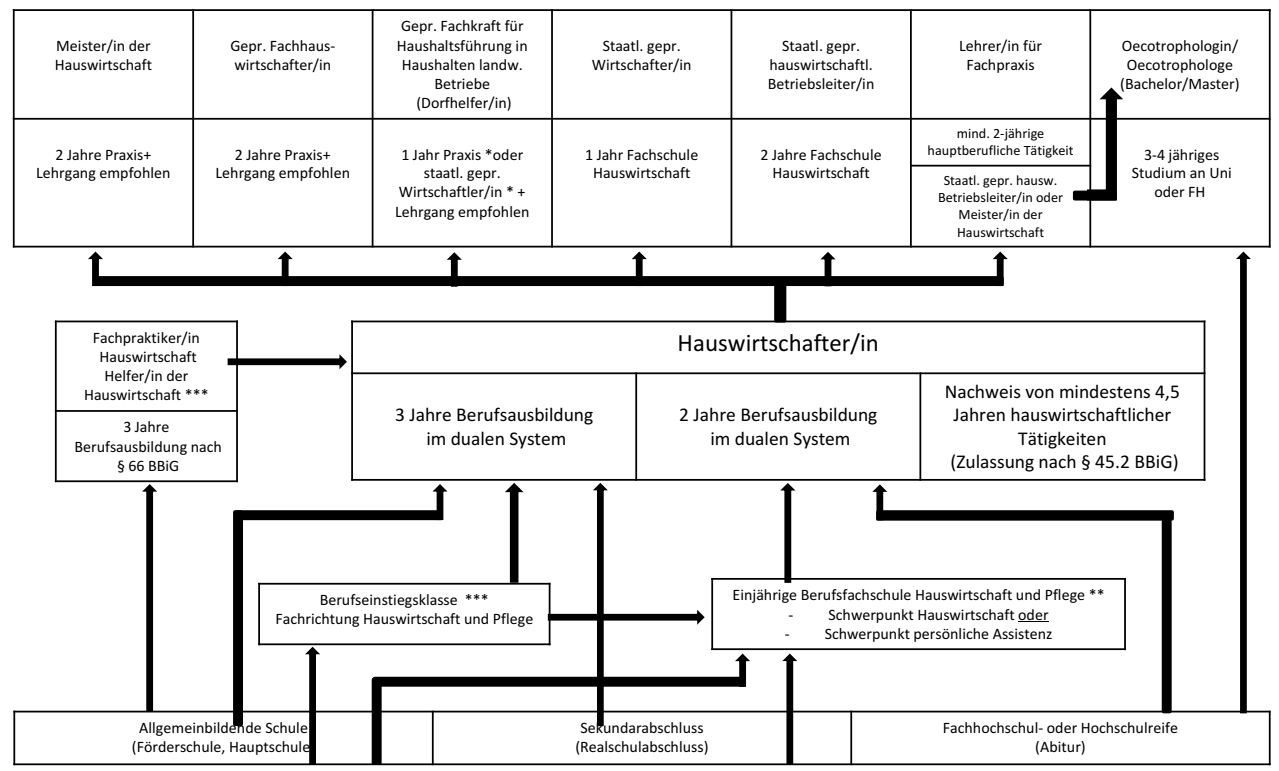

* Davon mindestens 6 Monate Haushaltsführung im landw. Betrieb

** Eingangsvoraussetzungen mind. Hauptschulabschluss anschl. Besuch der zweijährigen Berufsfachschule - Fachrichtung Ernährung und Hauswirtschaft - möglich

*** Allgemeinbildender Schulabschluss ist nicht Eingangsvoraussetzung

Abb. 2: Übersicht über Aus- und Weiterbildung in der Hauswirtschaft (Quelle: Landwirtschaftskammer Niedersachsen, o.J $)^{1}$

Aufbauend auf der Fachkraftstufe gibt es Fortbildungsmöglichkeiten an Fachschulen oder sonstige berufsbegleitende Qualifizierungen (z.B. Berufsabschluss als Meisterin/Meister, als Staatlich geprüfte Wirtschafterin/Staatlich geprüfter Wirtschafter) (Wiener, Winge \& Zetsche, 2014, S. 29).

Bei Vorliegen der Hochschulzugangsberechtigung besteht darüber hinaus die Möglichkeit eines Oecotrophologie-Studiums an einer Hochschule sowie der Wahl 


\section{Profilschärfung der Hauswirtschaft}

eines ,hauswirtschaftsnahen“ Studienfaches im Rahmen eines Lehramtsstudiums für die verschiedenen Schulformen und -stufen (Brutzer, 2014, S. 96 f.).

\subsection{Strukturelle Merkmale der Ausbildungssituation}

Gemeinsam ist den hauswirtschaftlichen Aus- und Weiterbildungsgängen ein Frauenanteil von fast $90 \%$. Es hat sich über die Jahre hinweg eine „starre“ Verbindung zwischen Geschlecht und Berufswahl verfestigt. Bei aller berechtigten Diskussion hierzu ist entscheidend, dass mit der geschlechtlichen Konnotation keine negativen oder positiven Diskriminierungen verknüpft sind oder, wenn vorhanden, ihnen klar und deutlich entgegen getreten wird (Kettschau, 2002). Dazu gehört auch die teilweise immer noch bestehende Schwierigkeit, hauswirtschaftliche, pflegerische oder erzieherische Aufgaben als berufsförmig organisierte, qualifizierte und gesellschaftlich hoch zu schätzende Arbeit anzusehen. Vielmehr werden diese Aufgaben als Teil einer „Frauenrolle“ (oder fortschrittlich „Elternrolle“) dem privaten Leben zugeordnet. Die Grenzen sind in der Tat kaum klar zu ziehen, sondern stetig Gegenstand gesellschaftlicher Veränderungs- und Aushandlungsprozesse. Wichtig ist, dass da wo Aufgaben aus dem ,privaten“ in den ,gesellschaftlichen“ Raum übergeleitet werden, die damit verbundene notwendige Professionalisierung ermöglicht und gestaltet wird.

Als wesentliches strukturelles Problem der hauswirtschaftlichen Ausbildungen ist das Verhältnis zwischen der Regelausbildung auf der Fachkraftstufe und der Fachpraktikerinnen- und Fachpraktikerausbildung zu nennen. Diese hat sich seit den 1990er Jahren stetig in Richtung auf eine Überzahl der Fachpraktikerinnen- und Fachpraktikerausbildungen verändert, was vor allem daran liegt, dass sich die Zahl der Auszubildenden im Beruf Hauswirtschafterin/Hauswirtschafter seit $1995 \mathrm{mehr}$ als halbiert hat und nunmehr bei ca. 3500 über alle Ausbildungsjahre liegt (siehe Tabelle 2). Durch diese Entwicklung konnte die sozialintegrative Aufgabenstellung der Berufsausbildung für junge Menschen mit Behinderungen gegenüber der beruflich-inhaltlichen Profilierung des Berufsbildes in den Vordergrund treten und die öffentliche Wahrnehmung und fachbezogene Diskussion teilweise dominieren.

Hinsichtlich der schulischen Lernausgangslagen bei Eintritt in die Ausbildung verwundert es nicht, dass rund $45 \%$ der Zugänge in die Fachpraktikerinnen- und Fachpraktikerausbildung ohne regulären Schulabschluss erfolgen (BIBB, 2017, S. 147). Fast $60 \%$ haben vor Aufnahme der Ausbildung schulische und/oder betriebliche Maßnahmen des Übergangssystems durchlaufen (siehe Tabelle 2). Ob allerdings diese Ausbildungen dem Auftrag des Berufsbildungsgesetzes gerecht werden können, d.h. berufliche Handlungskompetenz zu entwickeln und Anschlussperspektiven in den (ersten) Arbeitsmarkt eröffnen, ist Gegenstand offener Diskussionen. Im Sinne einer inklusiven Bildung wird es verstärkt darum gehen, allen Menschen Zugang zu anerkannten Ausbildungen zu ermöglichen. 


\section{Profilschärfung der Hauswirtschaft}

Dem hauswirtschaftlichen Berufssektor kommt hier auch zukünftig eine große Verantwortung zu. In den Berufsbildungswerken, Berufsverbänden und zuständigen Stellen sind unter der Zielsetzung inklusiver Bildung und gesellschaftlicher Teilhabe Konzepte fort- und neu zu entwickeln. Gerade in der Hauswirtschaft liegen hierzu umfangreiche Erfahrungen und Vorarbeiten vor, die eine Grundlage für eine tragende Rolle bei der inklusiven Berufsbildung sein können.

Bei der Ausbildung zur Hauswirtschafterin/zum Hauswirtschafter handelt es sich um einen regulären dualen Ausbildungsgang nach dem Berufsbildungsgesetz. Vergleicht man die bildungsstatistischen Daten mit denjenigen anderer nahe stehender Ausbildungsberufe, so sind keine sehr gravierenden Unterschiede festzustellen: das Gros der Auszubildenden (zwischen etwa 45 und 55\%) besitzt den Hauptschulabschluss, knappe $30 \%$ verfügen über einen Realschulabschluss, die „unteren“ (= ohne Hauptschulabschluss) und „oberen“ Ränder (= mit Fachhochschulreife oder allgemeiner Hochschulreife) sind jeweils mit deutlich unter $10 \%$ vertreten (siehe Tabelle 2).

Tab. 2: Merkmale von Auszubildenden und Ausbildungserfolg in der Hauswirtschaft (Quelle: eigene Berechnungen nach „Datensystem Auszubildende" des BIBB, 2017, Datenblätter: Hauswirtschafter/-in, Berufe für Menschen mit Behinderung in der Hauswirtschaft)

\begin{tabular}{|c|c|c|c|c|c|c|c|c|c|c|c|c|c|c|c|c|c|}
\hline & \multirow{2}{*}{ 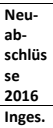 } & \multicolumn{2}{|c|}{$\begin{array}{l}\text { Neuabschlüsse } \\
\%\end{array}$} & \multirow{2}{*}{\begin{tabular}{l|} 
Ein- \\
stiegs- \\
alter \\
Durch- \\
schnitt
\end{tabular}} & \multirow[t]{2}{*}{$\begin{array}{l}\text { ohne } \\
\text { HS \% }\end{array}$} & \multirow[t]{2}{*}{$\begin{array}{l}\text { HS } \\
\%\end{array}$} & \multirow[t]{2}{*}{$\begin{array}{l}\text { RS } \\
\%\end{array}$} & \multirow{2}{*}{$\begin{array}{l}\text { FHR } \\
\text { AHR } \\
\%\end{array}$} & \multirow{2}{*}{$\begin{array}{l}\text { Vor- } \\
\text { herige } \\
\text { TN } \\
\text { BV/BG } \\
\%\end{array}$} & \multirow[t]{2}{*}{$\begin{array}{l}\text { Lö- } \\
\text { sungs- } \\
\text { quote }\end{array}$} & \multirow{2}{*}{$\begin{array}{l}\text { Er- } \\
\text { folgs- } \\
\text { quote }^{1}\end{array}$} & \multirow{2}{*}{$\begin{array}{l}\text { Aus- } \\
\text { zubil- } \\
\text { dende } \\
\text { insge- } \\
\text { samt } \\
2016\end{array}$} & \multicolumn{5}{|c|}{$\begin{array}{l}\text { Auszubildende insgesamt } \\
\text { 1997-2015 }\end{array}$} \\
\hline & & w & $\mathrm{m}$ & & & & & & & & & & 1997 & 2006 & 2008 & 2014 & 2015 \\
\hline Hauswirtschafter/in & 1008 & 91,6 & 8,4 & 20,3 & 4,5 & 56,5 & 36,0 & 6,2 & 32,7 & 31,4 & 84,4 & 2673 & 8124 & 6315 & 6159 & 3186 & 2940 \\
\hline $\begin{array}{l}\text { Fachpraktiker/in } \\
\text { Hauswirtschaft }^{2}\end{array}$ & 1314 & 87,9 & 12,1 & 19,3 & 45,9 & 52,1 & 2,1 & 0 & 59,4 & 27,2 & 94,6 & 3675 & 6027 & 6612 & 6219 & 4182 & 3933 \\
\hline
\end{tabular}

Quelle: eigene Berechnungen nach „Datenbank Auszubildende" BIBB, 2017, Datenblätter: Hauswirtschafter/-in, Berufe für Menschen mit Behinderung in der Hauswirtschaft

${ }^{1}=$ Die Erfolgsquote ist prüfungsteilnahmebezogen

$2=$ Beruf nach $\S 66$ BBIG; heute: „Berufe für Menschen mit Behinderung in der Hauswirtschaft"

\begin{tabular}{|l|l|}
\hline Legende Abkürzungen \\
\hline HS & Hauptschulabschluss \\
\hline RS & Realschulabschluss \\
\hline FHR/AHR & $\begin{array}{l}\text { Fachhochschulreife/Allgemeine } \\
\text { Hochschulreife }\end{array}$ \\
\hline TN BV/BG & $\begin{array}{l}\text { Teilnahme an Berufsvorbereitung } \\
\text { bzw. beruflicher Grundbildung }\end{array}$ \\
\hline
\end{tabular}

\section{Reformetappen im hauswirtschaftlichen Fachdiskurs}

Hauswirtschaftliche Themen und Entwicklungen werden in Deutschland in einer ausgeprägten Fachszene bearbeitet. Zahlreiche Fachorganisationen und Berufsverbände führen einen regen, intensiven und kontinuierlichen Diskurs zu aktuellen Aufgabenstellungen und fachpolitischen Ausrichtungen. Der Fokus liegt zumeist darauf, die Stärken der Hauswirtschaft zu betonen und letztlich die Zukunftsfähigkeit der Hauswirtschaft zu sichern. Zahlreiche wissenschaftliche Arbeiten analysieren Ent- 


\section{Profilschärfung der Hauswirtschaft}

wicklungslinien, Bedingungsfelder und Herausforderungen der beruflichen Bildung in der Hauswirtschaft und stellen Bezüge zur übergeordneten berufspädagogischen und berufspolitischen Debatte her. In der Zusammenschau lassen sich für die letzten Dekaden folgende Reformansätze exemplarisch aufzeigen (siehe Abbildung 3).

Die 1990er Jahre waren generell eine innovative Epoche der beruflichen Bildung. Mit der Einführung der Lernfeldsystematik verband sich eine konzeptionelle und didaktische Kehrtwende in der Grundarchitektur des schulischen beruflichen Lernens. Handlungsorientierung und mehr Befähigung zu selbstständigem Lernen waren auch die zentralen Begriffe bei der Reform der praktischen Ausbildung in den Betrieben. Die Novellierung der hauswirtschaftlichen Berufsausbildung im Jahre 1999 konnte sich auf berufspädagogische Forschungen und Modellversuche stützen (Projekt MOBS - Mobiler Haushaltsservice, u.a.; Friese, Thiessen, Schweizer \& Piening, 2000). Unter dem Schlagwort „Von der Verrichtungsorientierung zur Dienstleistungsorientierung“ vollzogen die neu entwickelten Ordnungsmittel den Anschluss an die aktuelle berufspädagogische Konzeptentwicklung. Statt feststehende Regeln für ihr Handeln anzuwenden, sollten die Auszubildenden nunmehr eine Situation weitgehend selbstständig einzuschätzen lernen und über den geeigneten Umgang mit Personen und Aufgaben verantwortlich - auf Basis ihres Fachwissens entscheiden. Personenorientierte Betreuungsaufgaben wurden gleichberechtigt neben den Versorgungsaufgaben zu Profilelementen der Fachbildung. Die Ausrichtung auf den Dienstleistungsgedanken mit den Stichworten Kundenorientierung, Personenbezug, individuelle Ausrichtung des Dienstleistungsangebotes erforderte große Anstrengungen bei den Akteuren der Ausbildung und wurde durch weitere Projekte und Modellversuche unterstützt.

\begin{tabular}{|c|c|}
\hline$>1996-1999$ & Mobiler Haushaltsservice // 1999 Neuordnung \\
\hline 1999-2002 & Dienstleistung im Lernortverbund \\
\hline 2006-2009 & $\begin{array}{l}\text { Kompetenzentwicklung für haushaltsnahe } \\
\text { Dienstleistungen }\end{array}$ \\
\hline 2009-2010 & $\begin{array}{c}\text { Berufe der Hauswirtschaft im Deutschen } \\
\text { Qualifikationsrahmen }\end{array}$ \\
\hline 2010-2013 & Nachhaltigkeitsorientiertes Rahmencurriculum \\
\hline 2013-2014 & $\begin{array}{c}\text { Hauswirtschaft als Spiegel gesellschaftlicher } \\
\text { Herausforderungen }\end{array}$ \\
\hline 2015 & Anstoß Neuordnungsprozess \\
\hline
\end{tabular}

Abb. 3: Themenschwerpunkte im hauswirtschaftlichen Fachdiskurs (Quelle: eigene Darstellung) 


\section{Profilschärfung der Hauswirtschaft |}

Der „Lernortverbund“ - Thema eines übergeordneten Modellversuchsprogramms der frühen 2000er Jahre (Euler, 2004) - bot den beteiligten Partnern aus der hauswirtschaftlichen Berufsbildung die Chance eigene Fragestellungen im Kontext der übergeordneten berufspädagogischen Forschung zu entwickeln (u.a. das Projekt DILL - Dienstleistungen im Lernortverbund; Friese, Piening \& Stremme, 2003). Lernen im Lernortverbund stellt sich in der hauswirtschaftlichen Berufsbildung mit ihrer typischen Struktur divergenter Kleinbetriebe als eine besondere Herausforderung dar.

Unter dem Stichwort „Kompetenzentwicklung“ öffnet sich der Blick stärker hin $\mathrm{zu}$ den jungen Menschen, die mit ihren je individuellen Lernvoraussetzungen und Lebensbedingungen jenseits starrer Systeme gefördert werden sollen. Mit der Implementierung der Qualifizierungsbausteine als Instrument der Berufsausbildungsvorbereitung ins BBiG (2005) wurden für jene jungen Menschen neue niedrigschwellige Zugänge eröffnet, auf Basis ausgewählter Inhalte anerkannter Ausbildungsberufe erste berufliche Handlungskompetenz zu entwickeln und gleichzeitig für das jeweilige Berufsfeld zu sensibilisieren. So wurde im Rahmen des Modellprojektes „BertHa - Kompetenzentwicklung für Haushaltsnahe Dienstleistungen" aufgezeigt, dass niedrigschwellige Zugänge einen wichtigen Beitrag leisten, junge Menschen an das hauswirtschaftliche Berufsfeld heranzuführen und für dessen Vielfältigkeit zu sensibilisieren (Friese \& Brutzer, 2010; Brutzer, 2014).

Die Einführung des DQR auf Bundesebene wurde durch zentrale Mittel unterstützt, so dass einige Berufsbereiche frühzeitig exemplarisch eine Umsetzung diskutieren konnten. Für den hauswirtschaftlichen Bereich initiierte die dgh in einer beispielhaften eigenen Anstrengung die Erarbeitung einer Umsetzung des DQR auf das hauswirtschaftliche Berufsgefüge (siehe Tabelle 1) (dgh, 2012). Auch hier zeigt sich der wichtige Beitrag zum Anschluss an übergeordnete Diskussionen - ermöglicht durch das ehrenamtliche Engagement von Expertinnen der unterschiedlichen Stufen und Sektoren hauswirtschaftlicher Ausbildung.

Das „Nachhaltigkeitsorientierte Rahmencurriculum für die Ernährungs- und Hauswirtschaftsberufe" war ein Modellversuch als Teil eines - sechs Einzelprojekte umfassenden - Förderschwerpunktes des BIBB zum Ende der UN-Dekade Bildung für eine nachhaltige Entwicklung. Als einziges beteiligtes Projekt aus der Ernährungs- und Hauswirtschaftsbranche oblag diesem Modellversuch die Aufgabe, die spezifischen nachhaltigkeitsbezogenen Fragestellungen und Herausforderungen der Bildung im Berufsfeld Ernährung und Hauswirtschaft aufzugreifen und einzelberufsübergreifende Lösungsvorschläge zu entwickeln. Diese sollten in Konzept, Methode und Ergebnissen nach Möglichkeit nicht nur im Berufsfeld Ernährung und Hauswirtschaft Anwendung finden, sondern darüber hinaus eine generelle Übertragbarkeit auf Prozesse beruflicher Bildung beinhalten. Die Abschlussveröffentlichung (Kettschau \& Mattausch, 2014) bietet eine detaillierte Analyse der Arbeitsprozesse in der Gemeinschaftsverpflegung als exemplarischem Handlungsfeld für die Ausbildungsbe- 


\section{Profilschärfung der Hauswirtschaft}

rufe Hauswirtschafterin/Hauswirtschafter, Koch/Köchin, Restaurantfachleute und Fachleute für Systemgastronomie. Das entwickelte Curriculum bezieht alle Aspekte der beruflichen Handlungskompetenz ein und zeigt deutlich die Verankerungsmöglichkeiten von Nachhaltigkeitskompetenz auf Basis bestehender Ordnungsmittel sowie den Weiterentwicklungsbedarf im Rahmen zukünftiger Neuordnungsverfahren auf.

Die vom Bundesministerium für Wirtschaft und Energie beauftragte Studie „Hauswirtschaft als Spiegel gesellschaftlicher Herausforderungen“ (Wiener, Winge $\&$ Zetsche, 2014) legt eine Analyse der Hauswirtschaft vor und durchleuchtet diese mit ihren unterschiedlichen Dienstleistungsfeldern und dem Fokus auf berufliche Aus- und Weiterbildung. Die vielfältigen Einsatzgebiete sowie die wachsenden Bedarfe hauswirtschaftlicher Dienstleistungen werden aufzeigt und die pflegebegleitende Hauswirtschaft im ambulanten und stationären Bereich wird als ein neues Handlungsfeld genannt. In der Studie werden Handlungsempfehlungen formuliert, wie sich der hauswirtschaftliche Berufsbereich zukünftig aufstellen sollte, um ein attraktives und zukunftsweisendes Profil aufzuweisen.

Der gegenwärtig diskutierte Neuordnungsprozess zielt auf die Anpassung der Ausbildung entlang aktueller Gegebenheiten, neuer hauswirtschaftlicher Tätigkeitsfelder und Anforderungen (Digitalisierung der Arbeit, Datenschutz, Nachhaltigkeit), die zielgenauer und inhaltlich aktueller auf die Zielgruppen und Kunden der Hauswirtschaft ausgerichtet werden sollen. Die Inhalte der Ausbildung sollen kompetenzorientiert abgebildet und an die aktuellen Erfordernisse des Arbeitsmarktes angepasst werden. Damit verbunden ist die Hoffnung, das Berufsfeld attraktiver und zeitgemäBer darzustellen, so dass wieder mehr Jugendliche, insbesondere auch leistungsstärkere junge Menschen, eine hauswirtschaftliche Ausbildung absolvieren.

Der vorliegende Eckdatenentwurf (Stand: 15.12.2017) stellt als Alternative zur Hauswirtschafterin/zum Hauswirtschafter die Berufsbezeichnungen „Fachkraft für hauswirtschaftliche Dienstleistung und Ernährung“ und „Fachkraft für hauswirtschaftliches Betreuungs- und Versorgungsmanagement" zur Diskussion. Die Ausbildungsdauer umfasst weiterhin drei Jahre. Davon soll in mindestens sechs Monaten der Ausbildung eine Schwerpunktsetzung erfolgen. Vorgeschlagen werden drei Dienstleistungsschwerpunkte: personenbetreuende, serviceorientierte sowie ländliche-agrarische Dienstleistungen. Weiterhin ist eine konventionelle Prüfung, aufgeteilt in Zwischen- und Abschlussprüfung, vorgesehen. Eine Berufsgruppenbildung mit anderen Berufen wird ausgeschlossen. Themen, die über die Standardberufsbildpositionen hinausgehen, z.B. Nachhaltigkeit und Verbraucherschutz, sollen in fachliche Berufsbildpositionen integriert werden.

Kritisch in der Fachszene wird u.a. die vorgeschlagene Berufsbezeichnung diskutiert, da sich hier nicht das vielfältige Berufsprofil der Hauswirtschaft widerspiegelt und der Ernährung zu viel Raum gegeben werden könnte. Auch bei den Schwerpunktsetzungen gibt es Kritik, da sich diese nicht an eindeutig abgrenzbaren Tätig- 


\section{Profilschärfung der Hauswirtschaft}

keitsfeldern orientieren. Es bleibt abzuwarten, wie sich die weiteren Diskussionen entwickeln.

Die aufgezeigten Reformetappen und Themenschwerpunkte zeigen deutlich eine Orientierung an berufspädagogischen, bildungspolitischen und teilweise auch gesellschaftspolitischen Linien und Diskussionen. Es werden Themen aufgegriffen und bearbeitet, die von Relevanz für eine Weiterentwicklung der beruflichen Bildung sind. Somit stellt sich die hauswirtschaftliche Fachszene in den Kontext übergeordneter Debatten, teilweise treibt sie diese aus eigenen Mitteln, teilweise nutzt sie die Chance, Querverbindungen herzustellen und ihre Anliegen mit anderen zu verknüpfen (wie z.B. bei den Themen Nachhaltigkeit, Frauenberufe, berufliche Bildung junger Menschen mit Förderbedarf). Wünschenswert und ein Beitrag zur weiteren Stärkung der Hauswirtschaft wäre es, wenn diese Querverbindungen selbstbewusst und systematisch weiter ausgebaut würden, und somit aus der kollegialen Zusammenarbeit mit benachbarten Berufsbereichen nachhaltig Anregungen und Stärkung gewonnen würden.

\section{Diskussion der Profiltreiber und Profilhemmer für die Ausbildung in der Hauswirtschaft}

Im vorliegenden Beitrag wurden eine Reihe von Profiltreibern und Profilhemmern für die Ausbildung in der Hauswirtschaft aufgezeigt (siehe zusammenfassend Tabelle 3), die im Folgenden diskutiert werden.

Ein Profiltreiber liegt darin, dass mit der Hauswirtschafterin/dem Hauswirtschafter ein dualer Kernberuf vorliegt, der eine grundständige und solide Ausbildung mit Blick auf die Anforderungen des Beschäftigungssegments bietet. Das Berufsbild ist sehr differenziert und vielfältig: Es reicht von Berufseinstiegsklassen/Berufsfachschulen über die Ausbildung zur Fachpraktikerinnen/zum Fachpraktiker, die duale Ausbildung zur Hauswirtschafterin/zum Hauswirtschafter, vielfachen Möglichkeiten sich weiterzubilden und der Möglichkeit ein hauswirtschaftsnahes Studium aufzunehmen. Diese vertikale Durchlässigkeit ist ein Positivum, welches in den benachbarten Berufen (z.B. Nahrungshandwerksberufe, Hotel- und Gesundheitsberufe) erst in jüngster Zeit durch neue Bachelorstudiengänge aufgebaut wurde.

Tab. 3: Profiltreiber und Profilhemmer der hauswirtschaftlichen Ausbildung im Überblick (Quelle: eigene Darstellung)

\begin{tabular}{l|l}
\hline Profiltreiber & Profilhemmnisse \\
\hline - Dualer Kernberuf & - Kontinuierlicher Rückgang der \\
- Vertikal differenziert und vielfältig & Auszubildendenzahlen \\
aufgebaut & - Sich verfestigender Überhang an \\
- Durchstiege und Aufstiegschancen & Fachpraktikerinnen- und \\
& Fachpraktikerausbildungen \\
& - Ungünstige Lernausgangslagen \\
\hline
\end{tabular}




\section{Profilschärfung der Hauswirtschaft}

\begin{tabular}{|c|c|}
\hline $\begin{array}{l}\text { - Generalistisches Profil } \\
\text { - Vielfältige Anschlüsse an Nachbarberufe }\end{array}$ & $\begin{array}{l}\text { - Schwächeres Kernprofil als } \\
\text { Nachbarberufe }\end{array}$ \\
\hline $\begin{array}{l}\text { - Vielfältige Einsatzgebiete durch } \\
\text { gesellschaftlich weiter wachsende Bedarfe } \\
\text { an hauswirtschaftlichen Dienstleistungen } \\
\text { - Entwicklungschancen }\end{array}$ & $\begin{array}{l}\text { - „Frauenberufe“ } \\
\text { - Image und Kompetenzzuschreibung } \\
\text { - Druck durch Beschäftigung } \\
\text { Ungelernter/Schwarzarbeit } \\
\text { - Berufsmotivation? }\end{array}$ \\
\hline $\begin{array}{l}\text { - Inkludierende Potentiale für Jugendliche } \\
\text { mit Förderbedarf }\end{array}$ & $\begin{array}{l}\text { - Abgrenzung der Fachpraktikerinnen- und } \\
\text { Fachpraktikerausbildung zur dualen } \\
\text { Regelausbildung }\end{array}$ \\
\hline $\begin{array}{l}\text { - Innovationsbereitschaft } \\
\text { - Nachhaltigkeitskompetenz }\end{array}$ & $\begin{array}{l}\text { - } \text { Missmatch zwischen komplexen } \\
\text { Anforderungen und Ausbildungsrealität } \\
\text { - Marktwert der Berufe }\end{array}$ \\
\hline
\end{tabular}

Demgegenüber verzeichnet die hauswirtschaftliche Erstausbildung strukturelle Schwierigkeiten mit Blick auf die Nachwuchsentwicklung. Insgesamt ist seit Mitte der 1990er Jahre ein kontinuierlicher Rückgang der Auszubildendenzahlen zu verzeichnen. Differenziert man die Zahl der Auszubildenden nach Fachpraktikerinnen- und Fachpraktikerebene und Fachkraftebene, dominiert erstere. Die damit verbundenen Herausforderungen, wie z.B. schwache Lernausgangslangen der Auszubildenden oder auch die Frage der Berufsmotivation wirken sich nicht günstig auf die Außenwahrnehmung aus und stellen die eigentliche Stärke der Hauswirtschaft in den Hintergrund.

Durch die stärkere Dienstleistungsorientierung der Hauswirtschaft hat sich das hauswirtschaftliche Aufgabenspektrum erweitert, denn es sind nun auch stärker betreuende und beratende Aspekte hinzugekommen. Die breit angelegte (generalistische) Ausbildung ermöglicht vielfältige Einsatz- bzw. Beschäftigungsmöglichkeiten (z.B. im betreuenden/pflegenden Bereich), vor allem im Nahtstellenbereich zu Betreuung und Pflege. Somit ergeben sich Anschlüsse an Nachbarberufe und eröffnen neue Formen der Zusammenarbeit, z.B. in multiprofessionellen Teams.

Gleichwohl kann bei fehlender Abgrenzung zu verwandten Berufen das Alleinstellungsmerkmal der Hauswirtschaft verwischen. Die sich daraus ergebenden Abgrenzungsprobleme und Konkurrenz sind wenig förderlich für die Hauswirtschaft, gerade in Zeiten zunehmender Spezialisierung bzw. im Hinblick auf OutsourcingTendenzen. Hinzu kommt, dass benachbarte Berufsgruppen teilweise einen höheren gesellschaftlichen Stellenwert genießen. Für viele junge Menschen scheint die Hauswirtschaft ein wenig attraktives Beschäftigungsfeld zu sein und der Berufswahl wird oftmals lediglich eine Überbrückungsfunktion in attraktivere Ausbildungen zugeschrieben. Nur für einen kleineren Teil der jungen Menschen ist die Hauswirtschaft die erste Wahl. Hier gälte es, durch Beobachtungen und Forschung heraus zu finden, welche Faktoren einen hauswirtschaftlichen Beruf in den Augen junger Berufssuchender attraktiv erscheinen lassen und diese gezielt auszubauen. 


\section{Profilschärfung der Hauswirtschaft |}

Die Hauswirtschaft ist einem zukunftsfähigen Beschäftigungssegment zuzuordnen, denn die Nachfrage an hauswirtschaftlichen Dienstleistungen ergibt sich aus

a) den demographischen Entwicklungen, d.h. der älter werdenden Gesellschaft und dem damit verbundenen Bedarf an Service, Versorgung und Betreuung;

b) der zunehmenden Erwerbstätigkeit von Eltern und der sich daraus ergebenden Nachfrage an haushalts- und familienunterstützenden Dienstleistungen,

c) der zunehmenden Nachfrage von Schulen und Kindertageseinrichtungen, z.B. unter den Stichworten Ganztagsbetreuung und Schulverpflegung;

d) den Megatrends Wellness, Gesundheit, Freizeiterleben, zu denen ebenfalls hauswirtschaftlich ausgerichtete Angebote platziert werden können.

Hieraus ergibt sich ein Bedarf an höher qualifizierten Fachkräften sowie grundständig qualifiziertem Service- und Assistenzpersonal. Mit dem breiten Spektrum an Aus- und Weiterbildungsmöglichkeiten bietet das Berufsfeld attraktive Anschluss- bzw. Aufstiegsmöglichkeiten auf unterschiedlichen Qualifikationsstufen. Die damit verbundenen Potenziale werden jedoch bislang aufgrund struktureller Schwächen nicht hinreichend ausgeschöpft. Zu nennen sind:

a) Beschäftigungsstruktur: Der überwiegende Anteil der Beschäftigten in der Hauswirtschaft ist ohne abgeschlossene Berufsausbildung tätig (Un- oder Angelernte). Dies trifft auf knapp 24\% der sozialversicherungspflichtig Beschäftigten zu und ist damit mehr als doppelt so hoch wie der Gesamtdurchschnitt aller sozialversicherungspflichtigen Beschäftigten (IAB, 2017). Ferner ist Teilzeit ein gängiges Beschäftigungsmodell: $61 \%$ der in der Hauswirtschaft tätigen Personen arbeiten in Teilzeit (Wiener, Winge \& Zetsche, 2014, S. 27). Ein Großteil der Dienstleistungen, vor allem im Privathaushalt, findet zudem in ungeschützten Beschäftigungsverhältnissen statt.

b) Zahlungsbereitschaft: Bewusstsein und Akzeptanz gegenüber professionell erbrachten und preislich entsprechend gestalteten hauswirtschaftlichen Dienstleistungen müssen in unserer Gesellschaft noch ausgebaut werden. Vergleicht man mit Eigenleistung oder "Schwarzarbeit" scheint fast jeder geforderte Preis, der eine Beschäftigung und Entlohnung zu angemessenen Bedingungen erlaubt, zu hoch.

c) Fachkraftmangel: Firmen, die hauswirtschaftliches Fachpersonal benötigen, klagen weithin über den Mangel an Fachkräften, so dass sie ihr Angebot einschränken müssen. Hier schließt sich der Kreis zu den 


\section{Profilschärfung der Hauswirtschaft}

Themen Attraktivität des Berufes, Motivation und Durchhaltefähigkeit der Auszubildenden sowie das Ausschöpfen der Potentiale aller Auszubildenden.

d) Angebotsformen: Angebote hauswirtschaftlicher Services einschließlich ihres Preis-/Leistungsverhältnisses, ihrer Qualität und den Vertragsbedingungen, scheinen bislang nicht breit und offensiv genug auf Märkten vertreten zu sein bzw. kommunikativ dargestellt zu werden. Der durchaus bereits entwickelte Markt für die Speisenversorgung (z.B. Lieferdienste, örtliche Kleinanbieter, Speisenversorgung außer Haus) lässt die Möglichkeiten auch für andere hauswirtschaftliche Services erahnen.

Ein Merkmal des deutschen Berufsbildungssystems ist dessen ganzheitlicher Ansatz, der neben wirtschaftlichen Interessen ebenso die Persönlichkeitsbildung des Einzelnen und seine Integration in die Gesellschaft zum Ziel hat. So kann es durchaus als Stärke gewertet werden, dass im hauswirtschaftlichen Fachpraktikerberuf wie auch teilweise im Hauswirtschaftsberuf im Vergleich zu anderen Berufen besonders viele junge Frauen mit ungünstigen Lernausgangslangen ausgebildet werden. Hierdurch leistet dieser Berufsbereich einen wichtigen Beitrag zur Persönlichkeitsentwicklung und zur sozialen und gesellschaftlichen Teilhabe dieser Personengruppen.

Dieser Profiltreiber ist ebenso profilhemmend. Der hohe Anteil an Ausbildungen mit Rehabilitationsziel (Fachpraktikerinnen- und Fachpraktikerausbildungen) kann zu einer Verlagerung der öffentlichen Wahrnehmung des vielseitigen, fachlich anspruchsvollen Berufsprofils der Hauswirtschaft weg und hin zu einem Bildungsgang mit schwerpunktmäßig ausgerichteten sozialpädagogischen Integrationsaufgaben führen. Zudem liegen Hinweise vor, dass das Arbeitslosigkeitsrisiko unter Fachpraktikerinnen und Fachpraktikern deutlich über dem der Hauswirtschafterinnen und Hauswirtschafter liegt (Wiener, Winge \& Zetsche, 2014, S. 35).

Der aufgezeichnete Entwicklungsprozess der letzten zwei Dekaden in der Hauswirtschaft belegt die hohe Innovationsbereitschaft des gesamten Berufsbereichs und die Fähigkeit, gesellschaftliche Trends aufzunehmen. Eine konsequente, systematische und nachhaltige Verwertung der Chancen, die sich aus den eigenen Initiativen ergeben, fehlt jedoch oftmals. Der zersplitterte Verbands- und Zuständigkeitsaufbau mit in Teilen konkurrierenden Strukturen erschwert eine gemeinsame Willensbildung. Innovationslinien, Modernisierungsbestrebungen und Megatrends zu nutzen und langfristig, konzeptionell in Verbindung zur hauswirtschaftlichen Entwicklung zu setzen, erscheint oft anrüchig und unterbleibt. 


\section{Profilschärfung der Hauswirtschaft}

\section{Perspektiven für den anstehenden Neuordnungsprozess}

Bezogen auf eine Neuordnung sind für die Weiterentwicklung des Ausbildungsberufes Hauswirtschafterin/Hauswirtschafter vier Innovationslinien maßgeblich in den Blick zu nehmen:

\subsection{Inklusion als Grundsatz}

Inklusion sollte als Grundsatz des Neuordnungsprozesses mitgedacht werden. Wenn man sich die bisherigen Auszubildenden der Hauswirtschaft anschaut, ist es unbedingt erforderlich, die Ausbildung inklusiv zu gestalten und die Möglichkeiten des BBIG zur Flexibilisierung auszuschöpfen. Konkret meint dies Überlegungen anzustellen, wie eine inklusive Berufsbildung pädagogisch-didaktisch sowie konzeptionell realisiert werden kann und im Zuge dessen die Entwicklung beruflicher Handlungskompetenz unterstützt werden kann. Experten inklusiver Berufsbildung monieren, dass die Fachpraktikerinnen- und Fachpraktikerausbildung dem inklusiven Grundgedanken nicht Rechnung trägt und eine exkludierende Maßnahme außerhalb des Regelsystems darstellt (Bylinski, 2015, S. 7). Aus diesem Grund sollte hauswirtschaftliche Ausbildung so gestaltet sein, dass berufliche Anschlüsse sowohl vertikal als auch horizontal für die Absolventinnen und Absolventen möglich sind. Die Diskussionen im Zuge der Modernisierungsbestrebungen der vergangenen Jahre hin zu einem flexibleren (Berufs)Bildungssystem bieten hierzu verschiedene Ansatzpunkte (z.B. Qualifizierungsbausteine oder gestufte Abschlussprüfung), die Ergebnisse des Innovationskreises Berufliche Bildung (z.B. Modularisierung in Form von Ausbildungsbausteinen) oder die im Zuge der Erarbeitung des DQR beschriebenen Kompetenzstufen zur Beschreibung des Learning Outcomes als Grundlage zur Herausarbeitung möglicher Anschlüsse und Durchstiege.

\subsection{Nachhaltigkeit als Grundlage hauswirtschaftlichen Handelns}

Nachhaltigkeit durch berufliches Handeln auf allen Ebenen anzustreben und umzusetzen, ist ein zentraler Beitrag zur Verwirklichung der Nachhaltigkeit als gesellschaftliches Ziel. In der UN-Dekade „Bildung für eine nachhaltige Entwicklung“ (2005-2014) wurden seitens des BIBB zwei Förderphase zur Implementation des Nachhaltigkeitsgedankens in die berufliche Bildung durchgeführt. Im Ergebnis steht fest: Die Berufsausbildung für eine nachhaltige Entwicklung sollte Teil eines modernen Berufsverständnisses sein. 2015 startete als Nachfolger der UN-Dekade das Weltaktionsprogramm Bildung für nachhaltige Entwicklung für fünf Jahre und eine weitere BBnE-Förderphase des BIBB. Das Motto der beruflichen Bildung dieses Weltaktionsprogramms lautet „Vom Projekt zur Struktur“, d.h. Nachhaltigkeit soll 


\section{Profilschärfung der Hauswirtschaft}

von Einzelprojekten zu einer strukturellen, curricularen Verankerung überführt werden. Das Projekt „Nachhaltigkeitsorientiertes Rahmencurriculum für die Ernährungsund Hauswirtschaftsberufe" (2010-2013) hat Anregungen für eine berufsfeldweite Verankerung des Nachhaltigkeitskonzeptes in der Fachrichtung Ernährung und Hauswirtschaft gegeben und Umsetzungschancen aufgezeigt. Der bildungspolitische und gesellschaftliche Diskurs für den Neuordnungsprozess sollte genutzt werden, um die strukturelle Verankerung der Nachhaltigkeitsbildung im Beruf der Hauswirtschafterin/des Hauswirtschafters voran zu treiben und das Berufsprofil entscheidend positiv zu prägen und zu modernisieren. Die Ansätze, die in der bisherigen Debatte zur Neuordnung vorliegen, zeigen noch nicht auf, dass dies schon ausreichend passiert ist.

\subsection{Kompetenzorientierung als Grundlage}

Mit Beschluss des Hauptausschusses des BIBB sollen seit 2015 alle neuen oder novellierten Ausbildungsordnungen in kompetenzorientierten Formulierungen gemäß dem DQR abgefasst werden. Hierzu bietet das BIBB als Hilfe einen entsprechenden Leitfaden an (BIBB, 2013). Durch die dgh wurden bereits in den Jahren ab 2009/2010 die wesentlichen Berufe der Hauswirtschaft in kompetenzorientierten Formulierungen vorgelegt (dgh, 2012). Im Rahmen der Neuordnung sind gestützt auf diese Vorgaben und Vorarbeiten das Berufsbild und der Ausbildungsrahmenplan entsprechen kompetenzorientiert anzulegen, um so Rückschlüsse auf Handlungen und Situationen zu ziehen, auf die Auszubildende vorbereitet werden sollen und in denen sich die erlangte Kompetenz bewähren muss. Zudem können mittels Kompetenzbeschreibungen Gemeinsamkeiten und Unterschiede der Hauswirtschaft zu anderen Bildungsangeboten im Berufsfeld Ernährung und Hauswirtschaft oder über das Berufsfeld hinweg herausgestellt werden. Sie dienen der Nachvollziehbarkeit sowie Transparenz und bieten die Möglichkeit der Abgrenzung und Präzisierung innerhalb und außerhalb des Berufsfelds.

\subsection{Digitalisierung in der Hauswirtschaft}

Gegenwärtig werden die Auswirkungen der Entwicklungen im Bereich Industrie 4.0 im Hinblick auf die sich daraus ergebenden Anforderungen und Herausforderungen für Konzepte und Curricula in der beruflichen Bildung breit diskutiert. Dieser Diskussion darf sich die Hauswirtschaft nicht entziehen, denn Fragen der Digitalisierung und deren Umsetzung in der beruflichen Bildung sind ein wichtiger Innovationsstrang für die Neuausrichtung und Profilbildung der Hauswirtschaft, auch wenn das Substituierungspotenzial im Vergleich zu anderen Berufszweigen momentan noch gering erscheinen mag. Obwohl zur Digitalisierung bisher noch keine Konzepte für die Hauswirtschaft vorliegen, fallen zahlreiche Anknüpfungspunkte für einzelne Handlungsfelder auf, seien es Versorgungsaufgaben in hauswirtschaftlichen Betrie- 


\section{Profilschärfung der Hauswirtschaft}

ben, hauswirtschaftliche Tätigkeiten in Freizeit, Gesundheit, Wellness oder Unterstützungsleistungen in Privathaushalten. Auch die Aufgaben eines modernen Qualitätsmanagements bedürfen einer elektronischen Systemunterstützung. Zum Einsatz kommen z.B. Onlineressourcen und Datenbanken zur Information über Rezepturen, Verfahrenstechniken, fachspezifische Wissensgrundlagen (z.B. Diätetik), Soziale Medien zur Nutzung von Marketingstrategien, elektronische Warenwirtschaftssysteme in Verbindung mit Einkauf, Speiseplanung sowie Lagerhaltung, BookingSysteme im Beherbergungs- und Beköstigungswesen, Kundenkommunikation mittels unterschiedlicher Kommunikationstechniken, Einsatz und Überwachung von elektronisch gesteuerten Assistenzsystemen in Privathaushalten etc. Die bereits bestehenden Verflechtungen der Hauswirtschaft mit der digitalen Welt und die zukünftige Dynamik zeichnen sich an dieser Stelle bereits ab. Auf die hieraus resultierenden Anforderungen sowie die damit verbundenen Herausforderungen sind Fach- und Führungskräfte vorzubereiten. In einem ersten Schritt sind hierzu wissenschaftliche Analysen und Bedarfserhebungen notwendig, um darauf basierend Konzepte auszuarbeiten.

Die aufgezeigten Innovationslinien sollten innerhalb der hauswirtschaftlichen „Szene“" weiterbearbeitet werden. Wichtig ist hier das Ziehen an einem Strang. Eine Novellierung alleine wird die aufgezeigten Profilhemmer nicht auflösen. Sie kann aber einen wichtigen Beitrag zur Profilbildung der Hauswirtschaft leisten.

\section{Anmerkung}

1) Es handelt es sich um eine Darstellung der zuständigen Stelle in Niedersachsen, die neben bundesrechtlichen ebenso landesspezifische Regelungen berücksichtigt. Deshalb treffen nicht alle dargestellten Details auf jedes Bundesland zu.

\section{Literatur}

BAG HW \& dgh - Bundesarbeitsgemeinschaft Hauswirtschaft \& Deutsche Gesellschaft für Hauswirtschaft e.V. (2012). Eckpunktepapier zur Weiterentwicklung des Ausbildungsberufes Hauswirtschafter/-in. Osnabrück. www.dghev.de/files/BAG-HW_dgh_Eckpunktepapier_Hauswirtschafterin.pdf BIBB - Bundesinstitut für Berufsbildung (2017). Datenreport zum Berufsbildungsbericht 2017. Bonn.

BIBB - Bundesinstitut für Berufsbildung (2017). Datensystem Auszubildende (DAZUBI). Datenblätter auf Basis der Berufsbildungsstatistik der statistischen Ämter des Bundes und der Länder (Erhebung zum 31. Dezember). www.bibb.de/dazubi 


\section{Profilschärfung der Hauswirtschaft}

BIBB - Bundesinstitut für Berufsbildung (2013). Leitfaden für die Entwicklung kompetenzorientierter Ausbildungsordnungen. Bonn.

Brutzer, A. (2014). Neue Qualifizierungsansätze für die berufliche Bildung. Konzepte für niedrigschwellige Qualifizierung am Beispiel Hauswirtschaft. Bielefeld: wbv.

Bylinski, U. (2015). Vielfalt als Ressource und Chance für gemeinsames Lernen und Entwicklung. In BIBB - Bundesinstitut für Berufsbildung (Hrsg.), Wege zur Inklusion in der beruflichen Bildung. Wissenschaftliche Diskussionspapiere des Bundesinstituts für Berufsbildung (S. 7-30), 62. Bonn.

dgh - Deutsche Gesellschaft für Hauswirtschaft e. V. (2012). Die hauswirtschaftlichen Berufe im Deutschen Qualifikationsrahmen. Osnabrück.

Euler, D. (Hrsg.) (2004). Handbuch der Lernortkooperation. Bd. 1: Theoretische Fundierung (S. 428-445). Bielefeld: Bertelsmann.

Freese, E. \& Toben-Vollmer, E. (2005). Hauswirtschaft nach Lernfeldern. Kommunizieren und Betreuen. Hamburg: Handwerk und Technik.

Friese, M. (2010). Didaktisch-curriculare Aspekte für Fachrichtungen und Fachrichtungsbereiche personenbezogener Dienstleistungsberufe. In J.-P. Pahl \& V. Herkner (Hrsg.), Handbuch Berufliche Fachrichtungen (S. 311-327). Bielefeld: wbv.

IAB - Institut für Arbeitsmarkt- und Berufsforschung (Hrsg.) (o. J.). Berufe im Spiegel der Statistik. Nürnberg. http://bisds.infosys.iab.de/bisds/faces/Start.jsp

Friese, M. \& Brutzer, A. (2010). Schlussbericht der wissenschaftlichen Begleitung zum Modellprojekt BertHa „Kompetenzentwicklung für haushaltsnahe Dienstleistungen. Berufliche Qualifizierung - anerkannte Zertifizierung“. Gießen (unveröffentlicht).

Friese, M., Piening, D. \& Stremme, K. (2003). Abschlussbericht zum Modellversuch Dienstleistung im Lernortverbund (DILL) im BLK-Programm KOLIBRI „,Kooperation der Lernorte in der Berufsbildung “. Teil A: Wissenschaftiche Begleitung. In Ministerium für Schule, Wissenschaft und Forschung des Landes Nordrhein-Westfalen/Senator für Bildung und Wissenschaft des Landes Bremen (Hrsg.). Bremen

Friese, M., Thiessen, B., Schweizer, B. \& Piening, D. (2000). Abschlussbericht zur wissenschaftlichen Begleitung „Mobiler Haushaltsservice“ - ein innovatives Konzept für die Ausbildung und Beschäftigung von Hauswirtschaftern/innen $M O B S$. Senator für Bildung, Wissenschaft, Kunst und Sport, Freie Hansestadt Bremen (Hrsg.). Bremen.

Kettschau, I. (2002). Berufswahl und Berufschancen von Frauen in Frauenberufen. In M. Kampshoff \& B. Lumer (Hrsg.), Chancengleichheit im Bildungswesen (S. 183-196). Opladen: Leske und Budrich. https://doi.org/10.1007/978-3-663-09485-2_12 


\section{Profilschärfung der Hauswirtschaft |}

Kettschau, I. \& Mattausch, N. (2014). Nachhaltigkeit im Berufsfeld Ernährung und Hauswirtschaft am Beispiel der Gemeinschaftsverpflegung. Hamburg: Handwerk \& Technik.

Landwirtschaftskammer Niedersachsen (o.J.). Berufe der Hauswirtschaft in Niedersachsen. https://www.lwk-niedersachsen.de/download.cfm/dlc/1/file/ 866,8713.html

Wiener, B., Winge, S. \& Zetsche, I. (2014). Hauswirtschaft als Spiegel gesellschaftlicher Herausforderungen. Halle.

\section{Verfasserinnen}

Dr. ${ }^{\text {in }}$ Alexandra Brutzer

Justus-Liebig-Universität Gießen

Institut für Erziehungswissenschaft

Professur Berufspädagogik/Didaktik der

Arbeitslehre

Karl-Glöckner Str. 21B

D-35394 Gießen

E-Mail: alexandra.brutzer@erziehung.unigiessen.de

Internet: www.uni-

giessen.de/fbz/fb03/institute/ifezw/prof/bp
Prof. ${ }^{\text {in }}$ Dr. Julia Kastrup \& Prof. ${ }^{\text {in }}$ i.R.

Dr. Irmhild Kettschau

Institut für Berufliche Lehrerbildung

Fachhochschule Münster

Leonardo Campus 7

D-48149 Münster

E-Mail: kastrup@fh-muenster.de kettschau@fh-muenster.de

Internet: www.fh-muenster.de/ibl/ 